\title{
The preventive effects of asperuloside administration on dextran sodium sulfate-induced ulcerative colitis in mice
}

\author{
Keiichi Hiramoto $^{1 *}$, Kenji Goto ${ }^{1}$, Hidehisa Sekijima ${ }^{1}$, Kazuya Ooi ${ }^{1}$, Tetsuya Hirata ${ }^{2}$ and Takahiko Fujikawa ${ }^{1}$ \\ ${ }^{1}$ Department of Pharmaceutical Science, Suzuka University of Medical Science, Mie 513-8670, Japan \\ ${ }^{2}$ R\&D Center, Kobayashi Pharmaceutical Co., Ltd. 1-30-3 Toyokawa, Ibaraki, Osaka 567-0057, Japan
}

\begin{abstract}
Eucommia ulmoides leaf extract (ELE) is a widely used Chinese medicine; asperuloside is the active substance in ELE and has anti-obesity properties. This study examined the effects of asperuloside administration on dextran sodium sulfate (DSS)-induced ulcerative colitis. We produced a mouse model of ulcerative colitis by administering DSS for 5 days. Asperuloside $(160 \mathrm{mg} / \mathrm{kg})$ was also orally administered concomitantly with the DSS for the initial 5 days. DSS-induced ulcerative colitis was ameliorated after administration of asperuloside. Blood levels of interleukin (IL)-6, tumor necrosis factor (TNF)- $\alpha$, histamine, acetylcholine (ACh), and reactive oxygen species (ROS) increased in DSS-treated mice. In contrast, blood histamine and ACh levels were lower in asperuloside + DSS-treated mice than those in mice administered DSS only; however, blood IL-6, TNF- $\alpha$, and ROS levels did not change. Although expression of the ACh receptor (AChR) increased in mast cells from DSS-treated mice, the expression decreased after administration of asperuloside. These results suggested that asperuloside administration is effective as a prophylaxis/treatment of DSS-induced ulcerative colitis in mice.
\end{abstract}

\section{Introduction}

Ulcerative colitis is an inflammatory disorder attributable to autoimmune disorders [1] or bacterial infections [2] and is characterized by inflammation of the colon tunica mucosa, with repeating periods of remission and recrudescence. Inflammation begins in the intestinal rectum near a fundament and spreads toward the colon, inducing loose bowels, bloody and mucous-containing stools, thermacogenesis, and weight loss $[3,4]$. Numerous animal models of ulcerative colitis have been used to elucidate the underlying mechanisms. Dextran sodium sulfate (DSS) can induce inflammation limited to the colon in mice, with symptoms resembling those observed in human ulcerative colitis [5]. Therefore, we used a DSS-induced ulcerative colitis mouse model to search for a corrective strategy to ameliorate symptoms.

Eucommia ulmoides leaf extract (ELE) is a Chinese medicine with various reported effects [6,7]. ELE contains several active substances, including geniposidic acid, asperuloside, and chlorogenic acid. Particularly, asperuloside, iridoid glucosides shows decreased ATP production in the WAT, accelerated fatty acid $\beta$-oxidation in the liver, increased use of ketone bodies and glucose in skeletal muscle, and may have increased non-shivering thermogenesis due to UCP1 expression in the BAT [8]. The bile acids secretion by asperuloside promotes these processes, followed by improving accumulation of the visceral fat and the exacerbation of blood fatty acid levels under the high fat diet $[9,10]$. As such, improvements in lifestyle-related diseases are expected following ingestion of asperuloside.

However, there are no reports regarding the effects of asperuloside on ulcerative colitis. In this study, we examined the effects of asperuloside on a DSS-induced ulcerative colitis mouse model.

\section{Materials and Methods}

\section{Animal experiments}

Specific-pathogen-free, 8-weeks old male Institute of Cancer Research (ICR) mice (SLC, Hamamatsu, Shizuoka, Japan) were used. These mice were housed under a 12-h light and 12-h dark cycle, a constant temperature of $23 \pm 1^{\circ} \mathrm{C}$, and $50 \pm 10 \%$ relative humidity. Mice were fed 5.0\% (W/V) DSS (molecular weight: 36,000-50,000 Da; MP Biomedical, Solon, OH, USA) in the drinking water for 5 consecutive days to induce colitis. The development of colitis was monitored in each mouse by measuring body weight and observing the condition of the feces. Fecal condition scores were determined using two parameters, including stool consistency $(0=$ normal; $1=$ soft; 2

Correspondence to: Keiichi Hiramoto, Ph.D., Department of Pharmaceutical Science, Suzuka University of Medical Science, 3500-3 Minamitamagakicho, Suzuka, Mie 513-8670, Japan, E-mail: hiramoto@suzuka-u.ac.jp

Key words: asperuloside, DSS-induced ulcerative colitis, acetylcholine, muscarinic receptor, histamine

Special Issue: Application to prophylactic pharmacology of food

Hirotaka Oikawa

Assistant professor

Department of Pharmaceutical Sciences

Suzuka University of Medical Science

Japan

Takahiko Fujikawa

Professor

Suzuka University of Medical Science

Japan

Received: November 20, 2017; Accepted: December 01, 2017; Published: December 06, 2017 
= very soft, but formed; 3 = liquid $)$ and fecal bleeding $(0=$ negative; 1 = faintly blue; 2 = moderately blue; 3 = dark blue; 4 = blood visible; determined via the guaiac paper test); the sum of these two parameters was considered to be the individual's disease activity score [11]. This study was carried out in strict accordance with the recommendations of the guide for the care and use of laboratory animals of Suzuka University of Medical Science (approval number: 34). All surgeries were performed under pentobarbital anesthesia, and all efforts were made to minimize suffering.

\section{Chemical treatment}

Asperuloside (Kobayashi Pharmaceutical, Osaka, Japan) was suspended in saline and administered orally on each day of the experiment [12]. The mice were divided into the following 3 groups: a control group (non-treatment), a DSS-administered group, and a DSS + asperuloside-administered group. There were 6 animals in each group.

\section{Isolation of tissue samples and blood}

The mice were euthanized, and a 1-ml blood sample was immediately collected from each mouse via cardiac puncture. Tissue samples were then isolated from the colon and fixed in phosphatebuffered saline (PBS) containing 4\% paraformaldehyde.

\section{Staining}

Colon specimens were cut into $5 \mu \mathrm{m}$-thick sections and subjected to immunostaining. We described the details of the staining method in our previous report [13]. Briefly, the specimens were incubated overnight at $4^{\circ} \mathrm{C}$ with primary antibodies, including goat polyclonal anti-mast cell tryptase (1:50; Santa Cruz Biotechnology Inc., Santa Cruz, CA, USA) and rabbit polyclonal anti-muscarinic acetylcholine receptor (mAChR; 1:100; Abcam, Cambric, MA, USA) antibodies. After staining, the specimens were incubated at $25^{\circ} \mathrm{C}$ for $2 \mathrm{~h}$ with fluorescein isothiocyanate-conjugated (FITC) anti-goat or tetramethylrhodamine isothiocyanate-conjugated (TRITC) anti-rabbit immunoglobulins (both used at 1:30; Dako Cytomation, Glostrup, Denmark). Protein expression was evaluated immunohistochemically with a fluorescent microscope.

Measurement of plasma interleukin-6 (IL-6), tumor necrosis factor-alpha (TNF- $\alpha$, histamine, acetylcholine, and reactive oxygen species (ROS) levels

Blood samples were taken from the heart on the fifth day after the start of the experiment. Plasma IL-6, TNF- $\alpha$, and histamine levels were determined using commercial enzyme-linked immunosorbent assays (ELISA; IL-6 and TNF- $\alpha$ : R\&D Systems, Minneapolis, MN, USA; histamine: Bertin Pharma, Montigny le Bretonneux, France) according to the manufacturer's instructions. We also measured plasma acetylcholine and ROS concentrations using assay kits (acetylcholine: Cell Biolabs, San Diego, CA, USA; ROS: STA-347; Cell Biolabs, Inc., San Diego, CA, USA) according to the manufacturer's instructions.

\section{Statistical analysis}

Data are presented as means \pm standard deviation. Results were analyzed using Excel 2010 software (Microsoft, Redmond, WA, USA). Differences between groups were evaluated via a one-way analysis of variance followed by Tukey's post hoc test using SPSS v.20 software (SPSS Inc., Chicago, IL, USA). Results were considered significant at $\mathrm{p}<0.05$.

\section{Results}

\section{Effects of asperuloside administration in DSS-treated mice}

Diarrhea and fecal bleeding were observed after DSS treatment in mice. The severity of disease was lower in the asperuloside + DSStreated mice than that in mice treated with DSS only (Figure 1D). DSStreatment also resulted in a drastic decrease in body weight and colon length. The average colon length in asperuloside + DSS-treated mice was longer than that in mice treated with DSS only (Figure 1A,B,C).

Effects of asperuloside administration on plasma levels of IL6 , TNF- $\alpha$ histamine, acetylcholine, and ROS in DSS-treated mice

We measured the plasma levels of IL-6, TNF- $\alpha$ histamine, acetylcholine, and ROS in the DSS-treated mice after 5 days of treatment. Plasma IL-6, TNF- $\alpha$ histamine, acetylcholine, and ROS levels were increased after DSS treatment. However, histamine and acetylcholine levels were lower in asperuloside + DSS-treated mice than those in mice treated with DSS only. Plasma IL-6, TNF- $\alpha$ and ROS levels in the asperuloside + DSS-treated mice did not differ from those in mice treated with DSS only (Figure 2).

\section{Activation of muscarinic acetylcholine receptors (mAChR) during colon disruption}

Mast cell tryptase expression increased in the colons of DSS-treated mice. Furthermore, we observed an increase in $\mathrm{mAChR}$ expression, as well as colocalization of these receptors with mast cell tryptases in DSStreated mice. Expression of these receptors was lower in asperuloside + DSS-treated mice than that in mice treated with DSS only (Figure 3).

\section{Discussion}

The present study demonstrates that asperuloside, found in ELEs, ameliorates the symptoms of DSS-induced ulcerative colitis. In addition, plasma levels of histamine and acetylcholine, as well as the expression of colon $\mathrm{mAChR}$ were reduced after asperuloside administration.

It has been reported that colitis symptoms in the DSS-induced ulcerative colitis mouse model are attributable to TNF- $\alpha$ [14], IL-6 [15], and oxidative stress [16]. In this study, alterations in TNF- $\alpha$, IL-6, and ROS levels were not observed after asperuloside administration, suggesting they are not directly affected by asperuloside treatment. In contrast, events occurring in mast cells indicate an important role for the onset of colitis [17]; although expression of mast cells following asperuloside treatment did not differ from that in mice treated with DSS only, histamine levels decreased. However, mechanisms underlying the inhibition of histamine secretion from mast cells after asperuloside treatment are unknown. Asperuloside affects the central nervous system [18]; therefore, these results may be attributable to various neurological effects. AChRs (nicotinic receptors (nAChR) and $\mathrm{mAChR}$ ) are expressed on the mast cells, and degranulation is induced when a ligand combines with these receptors. It has been suggested that ACh, which is an AChR ligand, is increased in DSS-treated mice, resulting in an increase in the secretion of histamine from mast cells $[19,20]$. In addition, it has been reported that the $\mathrm{mAChR}$ cholinergic signal transmission system is involved in mast cell activation [21]. Asperuloside administration decreases ACh content and the expression of mAChRs in mast cells of mice with DSS-induced ulcerative colitis. These findings suggest that asperuloside suppresses degranulation by inhibiting the expression of $\mathrm{ACh} / \mathrm{mAChR}$ in mast cells; therefore, we 
A
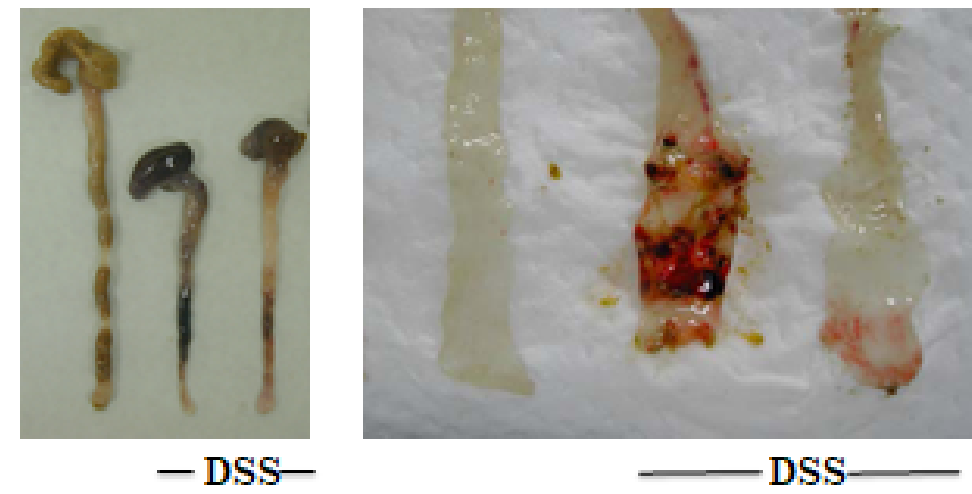

Asperuloside
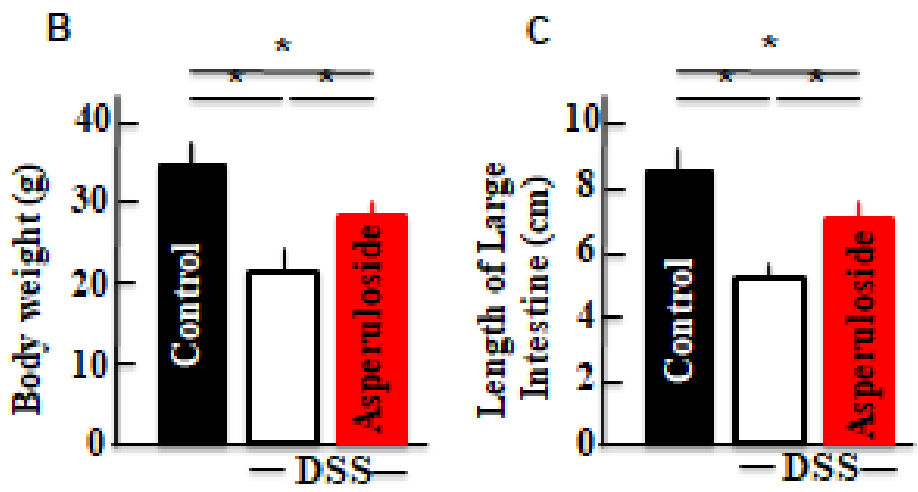

Asperuloside

D

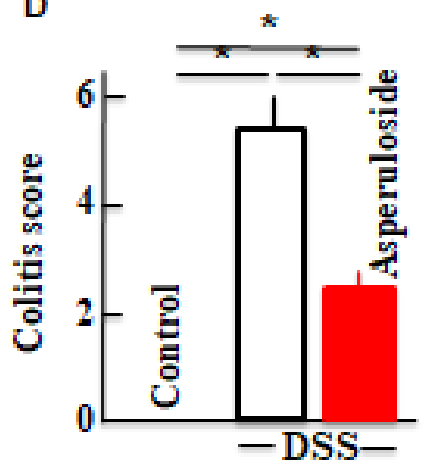

Figure 1. The effects of asperuloside administration on dextran sodium sulfate (DSS)-induced ulcerative colitis. Colitis scores (D) and animal body weights (B) are shown. The length of the large intestines $(\mathrm{A}, \mathrm{C})$ is shown. Values represent the means $\pm \mathrm{SD}$ derived from 6 animals. ${ }^{*} P<0.05$.
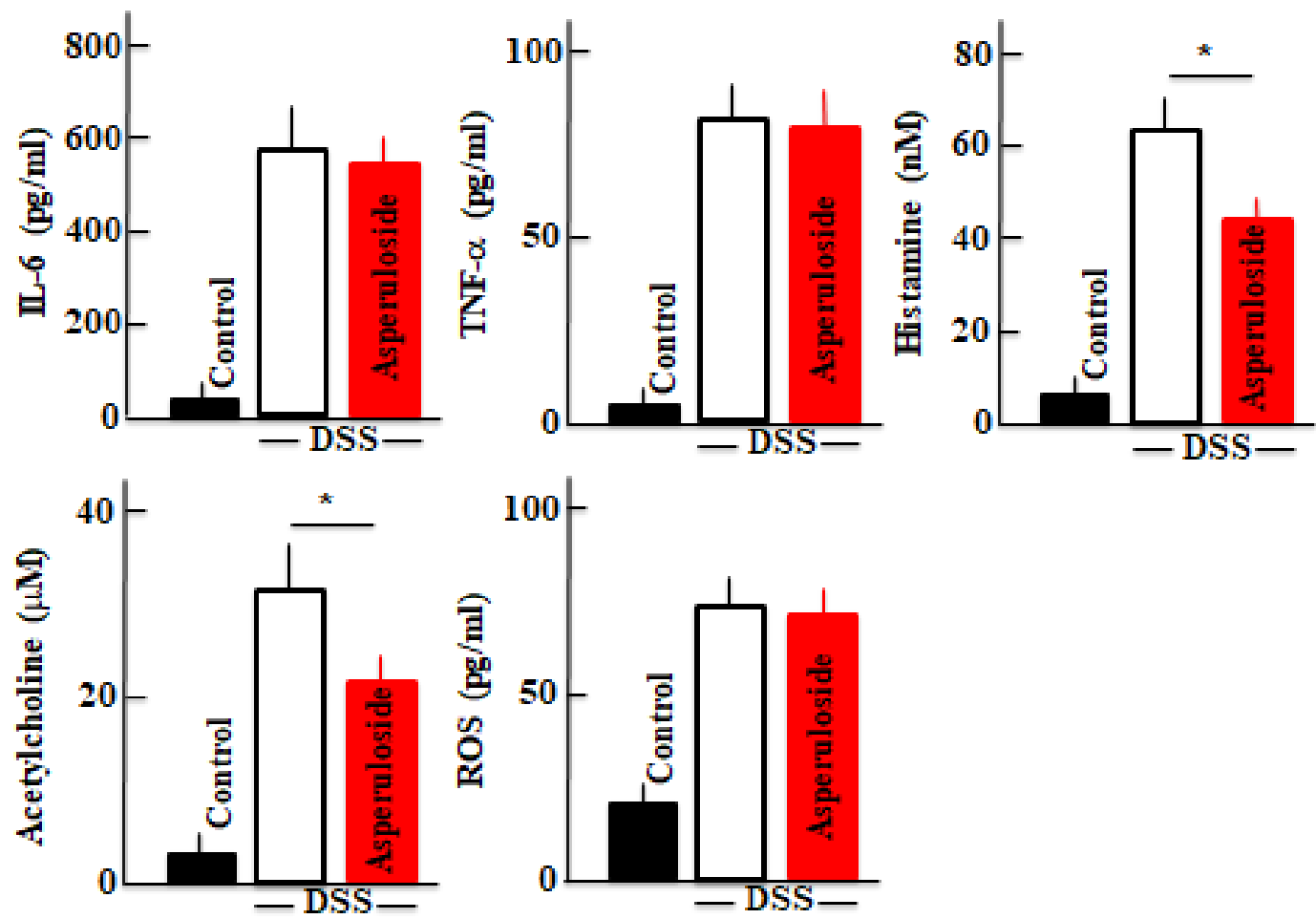

Figure 2. An analysis of plasma interleukin (IL)-6, plasma tumor necrosis factor-alpha (TNF- $\alpha$ ), plasma histamine, plasma acetylcholine (ACh), and plasma reactive oxygen species (ROS) concentrations after dextran sodium sulfate (DSS)- or asuperuloside + DSS-treatment is shown. Values represent the means \pm SD derived from 6 animals. $*, P<0.05$. 


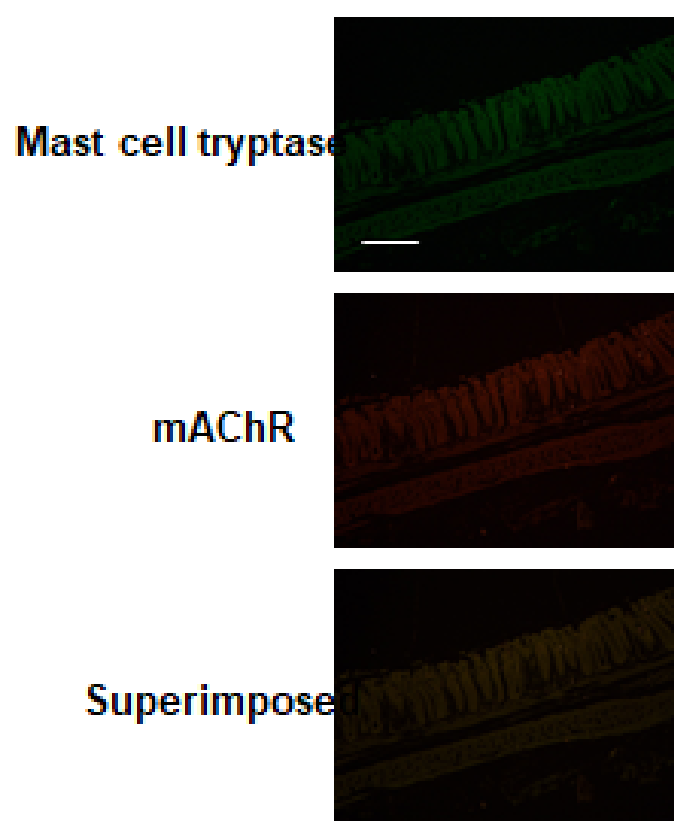

Control
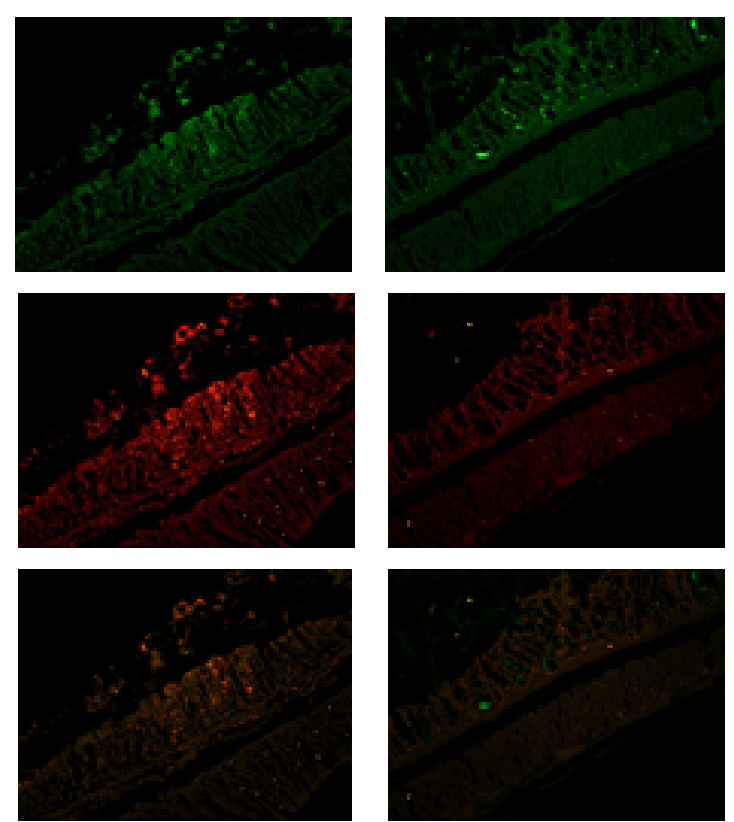

DSS

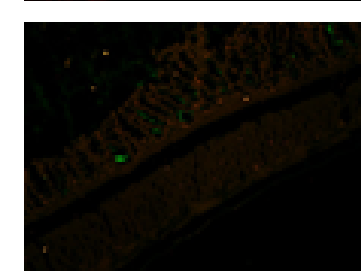

Asperuloside + DSS

Figure 3. The effects of asperuloside administration on mast cell tryptase proliferation and muscarinic acetylcholine receptors (mAChR) in the colon of dextran sodium sulfate (DSS)-treated mice are shown. We considered the relationship between mast cell tryptase and $\mathrm{mAChR}$ via double staining. One representative experiment performed on 6 animals is shown. Scale bar $=100 \mu \mathrm{m}$.

suggest that asperuloside ameliorates DSS-induced colitis. However, we do not fully understand the mechanisms by which ACh and mAChR are decreased, and further examination is necessary.

\section{Conclusion}

This study indicates the possibility that asperuloside targets signal transmission pathways through AChRs on mast cells and could prevent colitis. Asperuloside is an active substance contained in EUE, which is used as an auxiliary material for the prophylaxis and treatment of colitis and is known for its safety and low price. However, information regarding the effects and mechanisms of asperuloside is still lacking, and further reports are needed.

\section{Acknowledgments}

This study was supported by a research grant from the Japanese Society of Eucommia. The asperuloside samples from the Eucommia leaf extract used in this study were provided by Kobayashi Pharmaceutical Co., Ltd. (Osaka, Japan)

\section{Conflict of interest}

There are no conflicts of interest to declare.

\section{References}

1. Raedler A, Schreiber S (1992) Is ulcerative colitis an autoimmune disease? Dtsch Med Wochenschr 117: 1333-1338. [Crossref]

2. Strober W, Fuss IJ, Blumberg RS (2002) The immunology of mucosal models of inflammation. Annu Rev Immunol 20: 495-549. [Crossref]

3. Marcus R, Watt J (1969) Seaweeds and ulcerative colitis in laboratory animals. Lancet 2: 489-490. [Crossref]

4. Watt J, Marcus R (1973) Experimental ulcerative disease of the colon in animals. Gut 14: 506-510. [Crossref]

5. Randhawa PK, Singh K, Singh N, Jaggi AS (2014) A review on chemical-induced inflammatory bowel disease models in rodents. Korean J Physiol Rhamucol 18: 279288. [Crossref]
6. Deyama T, Nishibe S, Nakazawa Y (2001) Constituents and pharmacological effects of Eucommia and Siberian ginseng. Acta Pharmacol Sin 22: 1057-1070. [Crossref]

7. He X, Wang J, Li M, Hao D, Yang Y, et al. (2014) Eucommia ulmoides Oliv. ethnopharmacology, phytochemistry and pharmacology of an important traditional Chinese medicine. J Ethnopharmacol 151: 78-92. [Crossref]

8. Fujikawa T, Hirata T, Hosoo S, Nakajima K, Wada A, et al. (2012) Asperuloside stimulates metabolic function in rats across several organs under high-fat diet conditions, acting like the major ingredient of Eucommia leaves with anti-obesity activity. J Nutr Sci 1: 1-11. [Crossref]

9. Hirata T, Kobayashi T, Wada A, Ueda T, Fujikawa T, et al. (2011) Anti-obesity compounds in green leaves of Eucommia ulmoides. Bioorg Med Chem Lett 21: 17851791. [Crossref]

10. Hosoo S, Koyama M, Watanabe A, Ishida R, Hirata T, et al. (2017) Preventive effect of Eucommia leaf extract on aortic media hypertrophy in Wister-Kyoto rats fed a high-fat diet. Hypertens Res 40:546-551. [Crossref]

11. Nishimura T, Andoh A, Hashimoto T, Kobori A, Tsujikawa T, Fujiyama Y (2010) Cellobiose prevents the development of dextran sulfate sodium (DSS)-induced experimental colitis. J Cin Biochem Nutr 46: 105-110. [Crossref]

12. Fujikawa T, Hirata T, Wada A, Kawamura N, Yamaguchi Y, et al. (2010) Chronic administration of Eucommia leaf stimulates metabolic function of rats across several organs. Br J Nutr 104: 1868-1877. [Crossref]

13. Yokoyama S, Hiramoto K, Koyama M, Ooi K (2014) Skin disruption is associated with indomethacin-induced small intestine injury in mice. Exp Dermatol 23: 659-663. [Crossref]

14. Breese EJ, Michie CA, Nicholls SW, Murch SH, Williams CB, et al. (1994) Tumor necrosis factor alpha-producing cells in the intestinal mucosa of children with inflammatory bowel disease. Gastroenterology 106: 1455-1466. [Crossref]

15. Zhang H, Chen W (2017) Interleukin 6 inhibition by triptolide prevents inflammation in a mouse model of ulcerative colitis. Exp Ther Med 14: 2271-2276. [Crossref]

16. Schreiber O, Petersson J, Walden T, Ahl D, Sandler S, et al. (2013) iNOS-dependent increase in colonic mucus thickness in DSS-colitic rats. PLoS One 8: e71843. [Crossref]

17. Iba Y, Sugimoto Y, Kamei C (2002) Participation of mast cells in colitis inflammation induced by dextran sulfate sodium. Methods Find Exp Clin Pharmacol 24: 15-18. [Crossref] 
Hiramoto K (2017) The preventive effects of asperuloside administration on dextran sodium sulfate-induced ulcerative colitis in mice

18. Miyazaki S, Oikawa H, Nakamichi S, Hirata T, Yamasaki H, et al. (2017) Aroma of Eucommia leaf extract (ELE) causes reduced locomotor activity and increased NREM sleep, acting like the partially related factors of oral ELE's effects with locomotoractivity-dependent-increase in NREM- and REM-sleep. Global Drugs Ther 3: 1-8.

19. Radosa J, Dyck W, Goerdt S, Kurzen H (2011) The cholinergic system in guttate psoriasis with special reference to mast cells. Exp Dermatol 20: 677-679. [Crossref]
20. Blandina P, Fantozzi R, Mannaioni PF, Masini E (1980) Characteristics of histamine release evoked by acetylcholine in isolated rat mast cells. $J$ Physiol 301: 281-293. [Crossref]

21. Yokoyama S, Hiramoto K, Koyama M, Ooi K (2015) Impairment of skin barrier function via cholinergic signal transduction in a dextran sulphate sodium-induced colitis mouse model. Exp Dermatol 24: 779-784. [Crossref]

Copyright: $\odot 2017$ Hiramoto K. This is an open-access article distributed under the terms of the Creative Commons Attribution License, which permits unrestricted use, distribution, and reproduction in any medium, provided the original author and source are credited. 